\title{
Effects of neuromuscular exercises on q-angle, knee joint stability and ankle mobility of handball athletes.
}

\author{
Marcos Vinicius de Sousa Fernandes', Samuel Ribeiro Carvalho1, Wesley dos Santos Costa², Ivan Silveira De Avelar ${ }^{3}$, \\ Deise Aparecida de Almeida Pires Oliveira', Patrícia Espíndola Mota Venâncio', Viviane Soares ${ }^{4}$
}

\begin{abstract}
Background: The sport, when it comes to matters related to high performance, can lead the athlete to the development of risk factors for injuries. To minimize the effects of high load training, neuromuscular exercises may offer increased joint stability, thereby reducing risk factors, number of injuries, and minimizing the extent and severity of those that may arise. Objective: To evaluate the effects of neuromuscular exercises on Q-angle, knee joint stability and ankle mobility of high performance handball athletes. Methods: This is a controlled clinical trial conducted with 23 athletes (aged 18 to 37 years), randomly divided into intervention ( $n=12)$ and control $(n=11)$ groups. Athletes were evaluated with clinical tests for knee joint instability. The risk factors evaluated were ankle range of motion (ROM) in the dorsal flexion movement (tibia over the tarsus) and the Q-angle, which are factors related to ankle sprains and anterior cruciate ligament injury, respectively. A protocol of three neuromuscular exercises for lower limb was applied three times a week, lasting approximately six minutes for 12 sessions. Results: All athletes presented Q-angle higher than expected and in the group that underwent neuromuscular exercises there was a significant reduction in right Q-angle (pre: $23.7 \pm 2.9$ o; post: $20.2 \pm 2.5$ o, $p=0.016$ ) and left (pre: $22.5 \pm 2.5^{\circ}$; post: $20.1 \pm 1.8^{\circ}, p=0.021$ ). The range of motion of both ankles did not increase significantly, but there was an average increase of 2.19 in the right ankle and 2.40 in the left of the athletes who participated in the intervention group. Clinical tests indicated improvement in joint stability. Conclusion: A significant reduction in the right and left Q-angle was observed, as well as reducing the frequency of excessive anterior tibial translocation, which may be positively reflected in the knee joint stability.
\end{abstract}

Keywords: Knee Injuries; Proprioception; Neuromuscular; Handball.

\section{INTRODUCTION}

Currently, handball stands as one of the most popular sports in the world and is practiced in recreational, sporting and school environment ${ }^{(1)}$. However, it takes more than just a set of well-executed techniques and rehearsed plays. It is essential that there is a relationship between technique, tactics and the complexity imposed during the game intelligently ${ }^{(2)}$. For high performance, it is important to prioritize the specific peculiarities of competitive sport, also considering possible game situations, i.e., the factors of training, competition and regeneration should be systematized, so that all remain fully integrated. Despite its many benefits, high performance sports can develop many risk factors for injuries, and lead to pain and chronic injuries ${ }^{(3)}$. In handball, the most frequent injuries are in the joints, located mainly in the knee, ankle and shoulder as a result of typical movements of the sport such as changes of direction, jumping, falls after jumping and repetition of movements ${ }^{(4)}$. In addition, the literature points to the practice time as the main factor for the injury involvement, followed by the incorrect technique in the execution of the movements ${ }^{(3)}$. In this context, body control and neuromuscular balance are of paramount importance for handball practice, making it necessary that the athletes' functional capacities are more enhanced, as well as balance and motor coordination, which are basic components in the practice of the sport and contribute together for a better sports performance ${ }^{(5)}$.

Injury prevention is an incessant object of study, since it aims to improve osteomioarticular stability and increase the athlete's performance in sports. To this end, neuromuscular exercises act on functional limitations, besides improving the sensorimotor-motor balance ${ }^{(6)}$, and also used to reduce pain, improve functional performance and can be effective in reducing risk factors for knee injuries ${ }^{(6,7)}$. Given the above, 
there is a need to keep the lower limb joints fully stable because, due to the above movements, the joints, especially the knee, frequently suffer injuries. Therefore, it is essential to explore new strategies in the field of injury prevention in sport. Protocols with neuromuscular exercises are known to have beneficial effects on injury prevention, with improvement in risk factors after rehabilitation ${ }^{(8)}$. From this premise, the aim of the present study was to evaluate the effects of neuromuscular exercises on Q-angle, knee joint stability and ankle mobility of high performance handball athletes.

\section{METHODS}

\section{Participants}

This study is a randomized, controlled, open-label clinical trial composed of male handball athletes aged 18-37 years, belonging to the junior and adult categories of the main team from Handball of the city of Anapolis - GO, called Sport Handball of Anapolis (HANDESFA). Currently, the club has approximately 130 athletes, distributed from pre-child to adult (Figure 1). Only athletes who had been on the competition team for at least six months were included, and athletes with acute injuries less than 48 hours before the preassessment, pre-established osteoarticular pathologies and age below 18 years were excluded. Athletes were randomly assigned to the intervention group and the control group by drawing the training site, as the team had four training sites: Sports gym - UniEvangélica with 9 athletes, José Ludovico de Almeida State College with 8 athletes, College of Goiás Military Police - Cezar Toledo with 6 athletes and Auxilium College with 5 athletes. The draw was performed using an envelope and the places drawn for intervention group were Sports gym - UniEvangélica and José Ludovico de Almeida State College and the others were for control group. The research followed the guidelines of Resolution 466/2012 and the Declaration of Helsinki and was approved by the Research Ethics Committee of University Center - UniEVANGÉLICA with number 2.989.832/2018. All athletes signed the Informed Consent Form (ICF).

\section{Injury history and anthropometric measurements}

A semi-structured interview questionnaire was applied, containing information on age, comorbidities, duration and frequency of training per week and practice time. Body mass was measured with a digital scale (Filizola, model 2096 PP, São Paulo, Brazil), with a precision scale of $0.1 \mathrm{~kg}$ and a capacity of up to $150 \mathrm{~kg}$. The athletes were weighed with as little clothing as possible and stood with their backs to the scale display until the value shown on the scale display stabilized. Height was measured in meters $(\mathrm{m})$ using the stadiometer (Sanny, São Paulo, Brazil). The athletes remained in standing position, barefoot and with feet together, with their bodies erect and their backs to the stadiometer. After weight and height measurements, body mass index $\left(\mathrm{BMI}-\mathrm{kg} / \mathrm{m}^{2}\right)$ was calculated by dividing weight by height squared ${ }^{(9)}$

\section{Clinical trials}

The Apley, anterior and posterior drawer and drop tests were performed as a screening to evaluate knee and hip functionality, as well as to assess the presence of pain, and joint and ligament stability. Risk factors assessed were ankle range of motion (ROM) in dorsal flexion (tibial over tarsal) movement and $\mathrm{Q}$-angle, which are factors related to ankle sprains ${ }^{(10)}$ and with anterior cruciate ligament injury, respectively ${ }^{(11,12)}$. Measurements were performed by the same evaluator to avoid divergences.

\section{Range of motion}

Passive ankle dorsiflexion range of motion (ROM) and Q-angle were measured with a Lafayette Gollehon goniometer (model 01135, Australia). Passive ankle dorsiflexion ROM was measured with athletes sitting with popliteal region at the table edge and knees flexed at 90․ The goniometer axis was centered over the lateral malleolus and the arms were aligned with the fibular axis and the fifth metatarsal head ${ }^{(13)}$. Two measurements were taken and the mean used for analysis.

\section{Q-angle measurement}

The $Q$-angle (QA) is defined as the intersection of two lines, one that connects the anterior superior iliac spine to the center of the patella and another that connects the center of the patella to the tibial tuberosity ${ }^{(14)}$. Measurements were performed with athletes in supine position with feet together and an isometric quadriceps activation. All measurements were performed on both lower limbs and the reference values were 140 with standard deviation of $\pm 3^{\text {o(15) }}$. The neuromuscular exercises took place before each training after five minutes of warm-up trotting three times a week, lasting approximately six minutes, and a total of twelve intervention sessions were performed between November and December 2018. The exercises were performed after five minutes of warm up trot.

\section{Intervention Protocol}

The training was performed in circuit form, with 30 seconds of accomplishment and 10 seconds to change of station. Athletes should complete three laps on the circuit, with a 20 second break in each lap for rest. The execution was by independent effort, without the aid of the effort perception scale and without control of the hemodynamic variables. The selected exercises were adapted from the study perfomed by Achenbach et al. ${ }^{(10)}$ and consisted of squatting on unstable surface, single-legged squat and gluteus medius isometry. Squats on unstable surface were administered with body weight on two proprioception discs (brand Performance Pretorian, model BC TF VM, Rio de 
Janeiro, Brazil), with progression being performed with an increase in the support base - distance between the feet by 5 centimeters (Figure $2 \mathrm{~A}$ and $2 \mathrm{~B}$ ). The second was the single-legged squat and targeting, similar to the squat, in which the knee should follow the alignment of a marked band on the ground (Figure 2C). Progression was defined by base instability, first performed on the ground, then over a $20 \mathrm{~cm}$ step (brand HOORN, model EVA, Rio Grande do Sul, Brazil) (Figure 2D), and later on the jump (brand Oxer, model ECX45, Minas Gerais, Brazil) (Figure 2E). In the isometry of the gluteus medius muscle, the athlete was positioned in lateral decubitus, with alignment of the shoulder (humerus), hip (greater femoral trochanter), knee (fibular head) and ankle (lateral malleolus). The athlete performed a hip abduction at 450 with an elastic band from the mini bands kit (brand Proaction, model Látex band, São Paulo, Brazil) positioned at ankle level, remaining in position for 30 seconds, three times on each side. The progression of the exercise was made by the tension of the elastic bands (Figure 2F). Progressions were performed three times, once every four intervention sessions, and at the end, the athletes were reassessed for ankle ROM and Q-angle. For the control group, they were instructed to perform a preheating and, at the end of the training, to perform a global stretching.

\section{Statistical analysis}

Data were expressed as mean, standard deviation, frequencies, percentages and graphs. To test the normality of the data was used the Shapiro-Wilk test. For comparison of pre and post intervention results it was used Student's t-test for dependent samples (normal distribution) or Wilcoxon test (asymmetric distribution). Chi-square was used to verify the association of the groups with the anterior and posterior drawer test. Univariate analysis of variance (ANCOVA) verified the interaction between

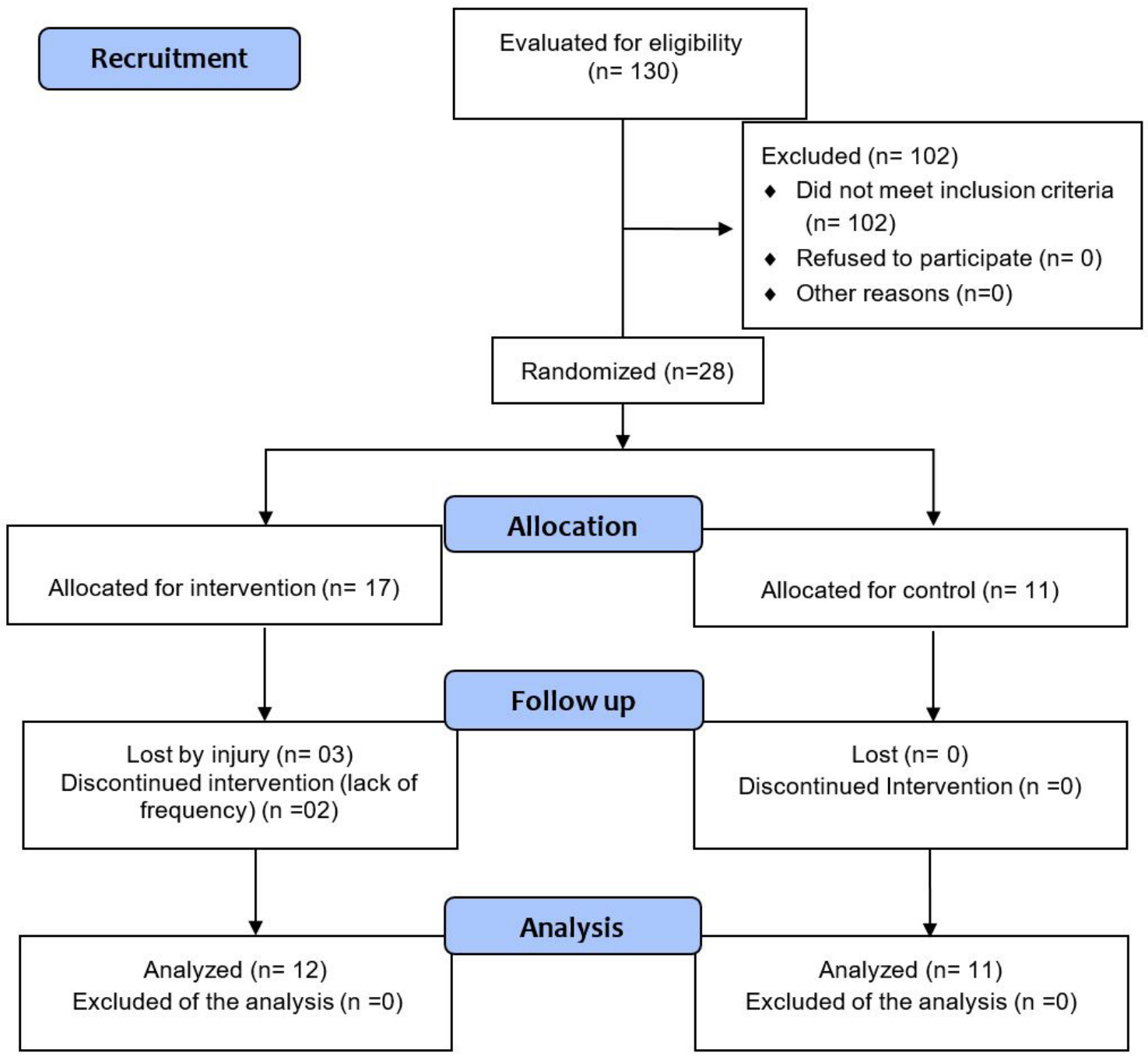

Figure 1. Study Flowchart 
intervention and control group. The $p$ value was $<0.05$. The software used for analysis was the Statistical Package Social Science (SPSS).

\section{RESULTS}

The data collection period was from November to December 2018. Twenty-eight athletes started the study, seventeen in the intervention group and eleven in the control group; however, five athletes were excluded from the intervention group because of injuries during the period in question and lack of frequency. There were no losses in the control group (Figure 1). Table 1 presents the sample characterization data. The age $(p=0.02)$ and practice time $(p=0.02)$ were higher in the intervention group. As for medications, two athletes make use, one in the intervention group (antacid for gastritis treatment) and one in the control group (anticonvulsant). The clinical drawer test was analyzed
Table 1 - Characterization of the sample.

\begin{tabular}{|c|c|c|c|}
\hline Variables & $\begin{array}{l}\text { Intervention } \\
\text { group } \\
\text { (n=12) } \\
\text { (SD) }\end{array}$ & $\begin{array}{l}\text { Control group } \\
\qquad \begin{array}{c}(n=11) \\
\text { (SD) }\end{array}\end{array}$ & $\mathbf{p}^{*}$ \\
\hline Age (years) & $24.00( \pm 5.61)$ & $19.18( \pm 2.04)$ & 0.02 \\
\hline Mass (kg) & $81.61( \pm 11.58)$ & $76.43( \pm 7.68)$ & 0.22 \\
\hline Height (cm) & $177.83( \pm 6.45)$ & $176.18( \pm 6.51)$ & 0.55 \\
\hline \multirow[t]{2}{*}{$\mathrm{BMI}\left(\mathrm{kg} / \mathrm{m}^{2}\right)$} & $25.85( \pm 3.75)$ & $24.69( \pm 2.98)$ & 0.42 \\
\hline & $\mathrm{n}(\%)$ & $\mathrm{n}(\%)$ & \\
\hline \multicolumn{4}{|l|}{ Medication use } \\
\hline Yes & $1(8.3)$ & $2(18.2)$ & 0.48 \\
\hline No & $11(91.7)$ & $9(81.8)$ & 0.48 \\
\hline Supplement use & $0(0)$ & $1(9.1)$ & 0.22 \\
\hline \multicolumn{4}{|l|}{ Training History } \\
\hline Training Frequency (n/week) & 5 & 5 & 0.99 \\
\hline Training Duration (min/week) & 450 & 450 & 0.99 \\
\hline Practice time (years) & $11.17(4.17)$ & 7.09 (3.89) & 0.01 \\
\hline
\end{tabular}

Note: $\mathrm{BMI}$ - body mass index. *Data for $\mathrm{p}<0.05$.

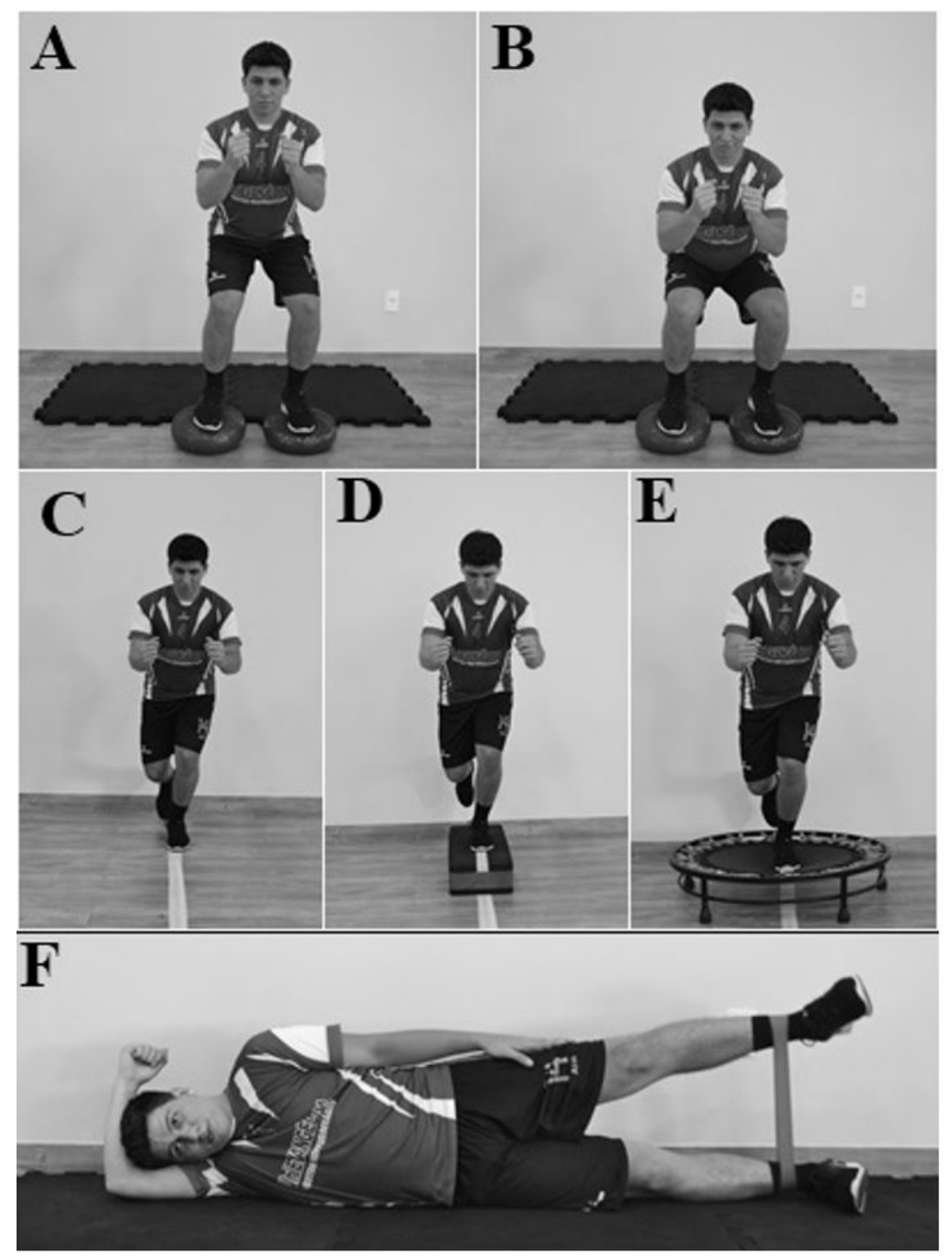

Figure 2. Neuromuscular exercises for lower limbs. Note: A and B: Squat on unstable surface. C, D and E: Single-legged squat with progressions. F: Position for gluteus medius isometry. 


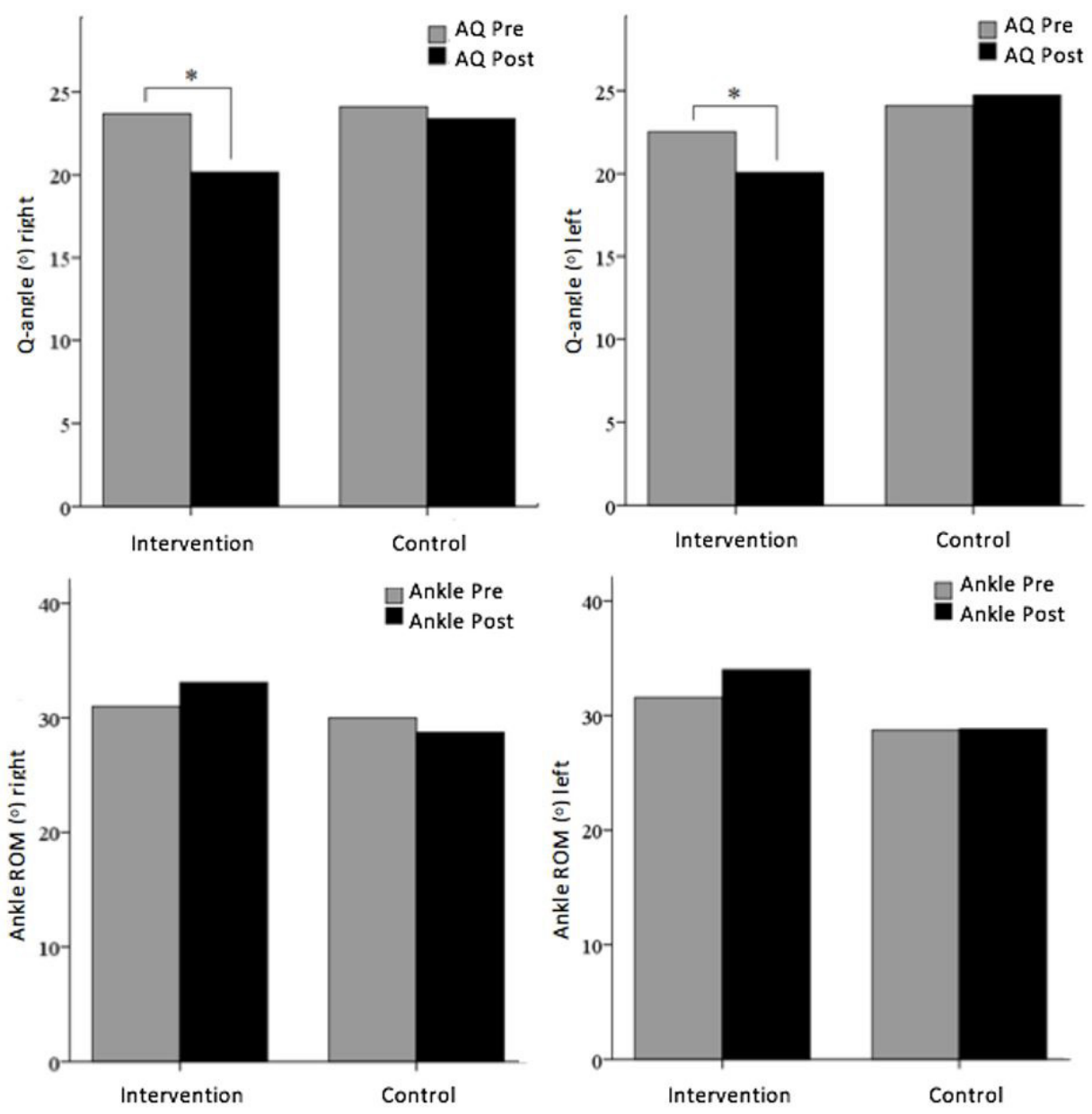

Figure 3. Pre and post measures of the $Q$-angle and range of motion of ankle/feet of intervention and control group of the left and right lower limb. Note: * $p<0,05$.

by frequency and clinical improvement was observed in the intervention group regarding tibial anteriorization (positive drawer test). However, there was no statistically significant result in the association between the groups. In the anterior cruciate ligament $(A C L)$ pre-evaluation, seven athletes presented excessive anterior tibial translocation in the right $(p=0.99)$ and left $(p=0.99)$ knees, indicating ligament laxity and, consequently, joint instability. After the intervention, the frequency was reduced for two athletes with positive drawer test in the right knee and three in the left. Regarding the posterior cruciate ligament $(\mathrm{PCL})$, there were no changes in the right $(p=0.38)$ and left $(p=0.22)$ knees. In the control group, four athletes presented positive test in the right and left knees and, at the end of the intervention period, the frequency increased for seven athletes with anterior displacement of the tibia in the left knee and nine in the right. In the case of PCL, initially three athletes presented positive test in the right knee and only one in the left. In the final evaluation, the frequency increased for six athletes with excessive anterior dislocation of the tibia in the right knee and five in the left, indicating marked joint instability.
The results regarding $Q$-angle and ankle/feet ROM pre and post neuromuscular exercises are shown in Figure 3. A significant reduction was observed when the right (pre: $23.7 \pm 2.9^{\circ}$; post: $20.2 \pm 2.5^{\circ}, p=0.016$ ) and left (pre: $22.5 \pm 2.5^{\circ}$; post: $20.1 \pm 1.8^{\circ}, p=0.021$ ) $Q$-angle was analyzed in the intervention group. The post-intervention mean still remained higher than predicted for males, but $02(17 \%)$ athletes in the intervention group had Q-angle within predicted for right knee. In the control group there was no improvement in $Q$-angle. Both groups did not show significant improvement regarding right $(p=0.37)$ and left $(p=0.23)$ ankle ROM, however, it is noteworthy that there was an increase in ankle mobility in the intervention group of 2.10 in the right and $2.4^{\circ}$ in the left.

\section{DISCUSSION}

The present study evaluated the effects of neuromuscular exercises on risk factors for injury of professional male handball athletes. After the intervention, the Q-angle of the intervention group had a significant reduction and two athletes presented the angle in the values predicted in the 
literature ${ }^{(15)}$. Knee injuries are always highlighted among the most common handball injuries, characterized by rupture of the cruciate and collateral ligaments and may be associated with $\mathrm{Q}$-angle changes. This is the angle formed by a line drawn by the patellar center and the tibial tuberosity, and another by the patellar center and the anterior superior iliac crest, which when elevated may increase the risk for knee injuries $^{(10,16)}$. Krosshaug et al. ${ }^{(17)}$ pointed to $\mathrm{Q}$-angle changes as one of the most important factors in increasing the risk of knee injury and several studies have shown the effects of precompetitive neuromuscular exercise and training on lower limb injury prevention ${ }^{(10,18)}$. A protocol similar to the one performed in the present study, lasting 20 minutes per session for 10 weeks twice a week, showed a reduction in knee valgus angles and, consequently, an improvement in leg stability, especially in athletes with severe valgus in preseason period $^{(18)}$. Furthermore, it is likely that a sports-specific training protocol with neuromuscular exercises may directly contribute to the prevention of knee injuries ${ }^{(10)}$. In contrast, Zebis et al. ${ }^{(19)}$ applied a protocol focused on improving body awareness and motor control of female handball athletes and achieved positive injury prevention results with improved knee valgus torque, but no Q-angle improvement. It is noteworthy that the protocol was performed with three exercises for the lower limbs, and lasting approximately 5 minutes for each exercise. In the present study, it was decided to perform circuit-shaped training, as the reduction in capsuloligamentous stability presented by the clinical tests in this study, promotes inadequate mechanoreceptor performance leading to a delay in muscle responses ${ }^{(20)}$. With these changes and the maintenance of 5 minutes of the same exercise as presented in the above study, it is suggested that mechanoreceptors may have fallen into fatigue.

Generally, training with neuromuscular exercises has beneficial effects on injury risk factors ${ }^{(21)}$. There is evidence in the literature of dynamic balance improvement only after six weeks of intervention using handball-adapted soccer exercises $^{(22)}$. In the present study, positive Q-angle results, which reflect high static valgus, occurred within four weeks of training. The prescribed exercises aimed to increase overall limb stability with emphasis on knees (joint stability) and gluteus medius, which is a hip stabilizer. Injuries at the Olympic level indicate that there is a higher frequency of lower limb injuries, especially in the knee, ankle and thigh, and the most common mechanisms are bruising and sprain $^{(23)}$. In a most recent study of Luig et al. ${ }^{(24)}$, analyzing the rate of injuries during the 2nd national handball league in Germany, there was greater frequency in the shoulders, hands and lower extremities and the most common mechanisms were also bruises and sprains.

The present study has some strengths, such as the existence of a standard reference control group for the intervention group, a training protocol composed of low cost exercises that meet the specificities of the modality and the effectiveness in reducing risk factors for sports injuries, specifically, the Q-angle that was the main object of the study. However, despite the reduction in $\mathrm{QA}$, the values remained high to those expected in the literature for males. The results suggest that the values would decrease even more if the protocol were part of the athletes training. Given the above, we suggest further research in the field of injury prevention with neuromuscular exercises and longer duration.

\section{CONCLUSION}

In conclusion, after intervention with neuromuscular exercises, a significant reduction in right and left $Q$ angle was observed, and it also reduces the frequency of excessive anterior tibial translocation (positive drawer test), characterizing a clinical improvement in knee joint stability. Regarding ankle mobility, clinical improvement was observed with an increase of $2.1^{\circ}$ in the right and $2.4 \%$ in the left; however, there was no statistically significant result.

\section{Acknowledgments:}

Laboratory of Evaluation and Intervention in Physiotherapy of the Centro Universitário de Anápolis, Anápolis (GO), Brazil.

\section{AUTHORS' CONTRIBUTIONS:}

MVSF participated in the study design, data collection and writing of the manuscript. SRC participated in the data collection. WSC participated in the writing and critical review of the manuscript. ISA was responsible for the critical revision of the manuscript. DAAPA critical review of the manuscript. PEMVcritical revision of the manuscript. VS participated in the study design, writing, writing improvement and critical revision of the manuscript.

\section{CONFLICT OF INTEREST:}

nothing to declare.

\section{AUTHORS DETAILS}

${ }^{1}$ Curso de Educação Física, Centro Universitário de Anápolis-UniEvangélica, Anápolis, Goiás, Brasil. ${ }^{2}$ Curso de Fisioterapia, Centro Universitário de Anápolis-UniEvangélica, Anápolis, Goías, Brasil. ${ }^{3}$ Curso de Educação Física, Faculdade da Polícia Militar de Goiás, Goiânia, Goiás, Brasil.

\section{REFERENCES}

1. Saavedra JM. Handball Research: State of the Art. J Hum Kinet. 2018; 63: 5-8

2. Menezes RP, Marques RFR, Nunomura M. O Ensino do Handebol na categoria infantil a partir dos discursos de treinadores experientes. Movimento. 2015; 21(2): 463-77.

3. Saragiotto BT, Di Pierro C, Lopes, AD. Risk factors and injury prevention in elite athletes: a descriptive study of the opinions of physical therapists, doctors and trainers. Braz J Phys Ther. 2014; 18(2): 137-43.

4. Freda FM, Rodrigues VD, Goulart RR. Prevalência de lesões esportivas em atletas de handebol de uma equipe profissional gaúcha. Revista Do Corpo: Ciências e Artes. 2015; 5(1): 1-5.

5. Hoffmeister AA, Rojas JM, Santos GMM, Filgueiras Junior N. Treinamento functional no Handebol. Rev. Magsul de Ed. Fís na Fronteira. 2016; 1(1): 173. 
6. Ageberg E, Roos EM. Neuromuscular exercise as treatment of degenerative knee disease. Exerc. Sport Sci. Rev. 2015; 43(1): 14-22

7. Bennell K, Dobson, F. Neuromuscular exercise for degenerative knees: can we optimize the treatment effect?. Exerc Sport Sci Rev. 2014; 43(1): 3-4.

8. Andersson SH, Bahr R, Clarsen B, Myklebust G. Preventing overuse shoulder injuries among throwing athletes: a cluster-randomised controlled trial in 660 elite handball players. Br J Sports Med. 2017; 51: 1073-80.

9. WORLD HEALTH ORGANIZATION (WHO). Diet, nutrition and the prevention of chronic diseases. Report of a Joint WHO/FAO Expert Consultation. Geneva. WHO Technical Report Series, v. 916, p. 23-50, 2003.

10. Achenbach L, Krutsch V, Weber J, Nerlich M, Luig P, Loose O et al. Neuromuscular exercises prevent severe knee injury in adolescent team handball players. Knee Surg Sports Traumatol Arthrosc. 2018; 26(7): 1901-8.

11. Olsen OE, Myklebust G, Engebretsen L, Bahr R. Injury Mechanisms for Anterior Cruciate Ligament Injuries in Team Handball: A Systematic Video Analysis. Am J Sports Med. 2004; 32(4): 1002-12.

12. Hewett TE, Myer GD, Ford KR, Heidt Jr RS. Colosimo AJ. Biomechanical Measures of Neuromuscular Control and Valgus Loading of the Knee Predic Anterior Cruciate Ligament Injury Risk in Female Athletes. Am J Sports Med. 2005; 33(4): 492-501.

13. Fong C-M, Blackburn T, Norcross MF, McGrant M, Padua DA. AnkleDorsiflexion Range og Motion and Landing Biomechanics. J Athl Train. 2011; 46(1): 5-10.

14. Weiss L, DeForest B, Hammond K, Schilling B, Ferreira L. Reliability of Goniometry-Based Q-Angle. PM R. 2013; 5: 763-8.

15. Wheeless CR. Textbook of Orthopaedics: Q angle of the Knee. 2012. Disponível em: http://www.wheelessonline.com/ortho/q_angle_of_the knee.

16. Higashi RH, Santos MB, De Castro GTM, Ejnisman B, Sano SS, Da Cunha RA. Lesões musculoesqueléticas em jovens atletas de handebol: Um estudo transversal. Fisioter. Pesqui. 2015; 22(1): 84-9.
17. Krosshaug T, Steffen K, Kristianslund E, Nilstad A, Mok KM, Myklebust $G$, et al. The vertical drop jump is a poor screening test for $A C L$ injuries in female elite soccer and handball players: a prospective cohort study of 710 athletes. Orthop J Sports Med. 2016; 44(4): 874-83.

18. Barendrecht M, Lezeman HCA, Duysens J, Smits-Engelsman BCM. Neuromuscular training improves knee kinematics, in particular in valgus aligned adolescent team handball players of both sexes. J Strength Cond Res. 2011; 25(3): 575-84.

19. Zebis MK, Andersen LL, Brandt M, Myklebust G, Bencke J, Lauridsen $\mathrm{HB}$, et al. Effects of evidence-based prevention training on neuromuscular and biomechanical risk factors for $\mathrm{ACL}$ injury in adolescent female athletes: a randomised controlled trial. Br J Sports Med. 2015; 50(9): 552-7.

20. Ellenbecker TS, Davies GJ, Bleacher J. 24 - Proprioception and Neuromuscular Control in Andrews JR, Harrelson GL, Wilk KE. Physical Rehabilitation of the Injured Athlete (Fourth Edition), W.B. Saunders, 2012.

21. Lauersen JB, Bertelsen DM, Andersen LB. The effectiveness of exercise interventions to prevent sports injuries: a systematic review and metaanalysis of randomised controlled trials. Br J Sports Med. 2013 Out 7; 48(11): 871-7.

22. Steib S, Zahn P, Eulenburg Cz, Pfeifer K, Zech A. Time-dependent postural control adaptations following a neuromuscular warm-up in female handball players: a randomized controlled trial. BMC Sports Sci Med Rehabil. 2016; 8:33.

23. Bere T, Alonso JM, Wangensteen A, Bakken A, Eirale C, Dijkstra HP, et al. Injury and illness surveillance during the 24th Men's Handball World Championship 2015 in Qatar. Br J Sports Med. 2015; 49: 1151-6.

24. Luig $P$, Krutsch $W$, Nerlich $M$, Henke T, Klein $C$, Bloch $H$, et al. Increased injury rates after the restructure of Germany's national second league of team handball. Knee Surg Sports Traumatol Arthrosc. 2018; 26(7): 1884-91. 